\title{
UPLCMS/MS-based Untargeted Metabolic Profiling Reveals Changes in the Plumbago auriculata Lam. Metabolome Following Heteromorphic Incompatibility System Interactions
}

\section{Di Hu}

Landscape Research Institute $₫$ Sichuan Agricultural University

\section{Wenji Li}

Landscape Research Institute $₫$ Sichuan Agricultural University

Suping Gao ( $\nabla$ gao_suping@sicau.edu.cn )

Landscape Research Institute, Sichuan Agricultural University

Ting Lei

Landscape Research Institute『Sichuan Agricultural University Ju Hu

Landscape Research Institute『Sichuan Agricultural University

\section{Ping Shen}

Landscape Research Institute囚Sichuan Agricultural University

\section{Yurong Li}

Landscape Research Institute『Sichuan Agricultural University

Jiani Li

Landscape Research Institute『Sichuan Agricultural University

\section{Research article}

Keywords: Plumbago auriculata, HetSI, non-targeted metabolomic analysis, pollen-stigma interaction, pin and thrum flowers, fluorescence microscopy, ESEM, UPLC-MS/MS

Posted Date: June 4th, 2019

DOI: https://doi.org/10.21203/rs.2.10039/v1

License: (9) (1) This work is licensed under a Creative Commons Attribution 4.0 International License. Read Full License 


\section{Abstract}

Background Heteromorphic self-incompatibility (HetSI), which is regulated by gametophytes, occurs in some species as a strategy to promote cross-pollination. Studies on HetSI are rare, and there is little evidence to establish the mechanism of HetSI. This research aimed to reveal metabolic changes occurring in HetSI. We used fluorescence microscopy as a tool to compare growth behavior in selfincompatible (SI) and self-compatible (SC) pollination in both pin and thrum flowers of Plumbago auriculata and to identify the ideal timepoint for sample collection for subsequent experiments. We also employed environmental scanning electron microscopy (ESEM) to evaluate intermorph structural differences in the pollen grain and stigmas in relation to heteromorphic self-incompatibility. Importantly, UPLC-MS/MS was applied in this study to identify metabolites, compare metabolic differences between pin and thrum styles and monitor metabolic changes in styles and SC and SI pollinations in the two type of flowers. Results The metabolites mainly included amino acids, flavonoids, glycosides/sugars, phenols, other organic acids, fatty acids/lipids, amines and alcohols. Surprisingly, high-energy nutrients such as amino acids, flavonoids, phenols alcohols and tricarboxylic acid cycle-related metabolites were found at higher levels in SI pollinations than in SC pollinations. Conclusion This result indicates that physiological changes in pollen-stigma interactions are different in pin, thrum styles and SC, SI pollinations and that energy deficiency may not be one of the reasons for HetSI.

\section{Background}

Heterostyly is a genetic polymorphism comprising distylous or tristylous mating types that differ in reciprocally arranged female and male reproductive organs and promote disassortative pollination via segregated pollen deposition onto pollinators [1-4]. Distylous species have two floral morphs: pin (stigma above anthers) and thrum (stigma below anthers) (Figure 1). According to Barrett [5], heterostyly is one of the strategies used to alleviate reproductive pressures and to promote cross-pollination for hermaphrodite flowers. To increase the outcrossing rate and promote the development of the entire species [1], heterostyly is always accompanied by self-incompatibility (SI) as a safeguard against self-fertilization and inbreeding depression [6], so-called heteromorphic self-incompatibility (HetSI).

Genetic information may be reflect in the metabolome [7], and a single metabolite might influence gene expression, protein stability and metabolic fluxes [8-11]. Ultra-high-performance liquid chromatography separation combined with quadrupole time-of-flight tandem mass spectrometry detection (UPLC-MS/MS) is a powerful technique for identifying metabolites in plants [12], and untargeted scanning is invaluable for identifying a large set of metabolites and understanding metabolic and physiological processes in plant physiology.

The self-incompatibility mechanism comprises homomorphic (HomSI) and hetermorphic (HetSI) selfincompatibility. However, only HomSI has been widely studied in terms of morphology, transcriptomes, proteomes, and metabolomes [13-17]. Indeed, studies on HetSI are rare, and there is little evidence to establish the metabolic mechanism of HetSI. 
The subfamily Plumbaginoideae consists of four genera, the largest of which contains approximately 20 species. Due to their range of variation, members of Plumbaginoideae provide an ideal system for investigating the mechanism of HetSI [18], and Plumbago auriculata (Plumbaginaceae) is included in this subfamily. This is the first study to assess morphological differences, identify metabolites and monitor metabolic changes in $P$. auriculata during different pollinations in an attempt to characterize biochemical changes that occur with different pollination combinations and to provide supplementary data for the HetSI mechanism.

\section{Results}

\subsection{Growth Behaviors of Pollen Tubes in Compatible and Incompatible Groups}

Pistils are composed of the stigma, style and ovary. A compatible fertilization includes successful pollenstigma recognition, pollen grain germination, pollen tube migration, and arrival at the ovary, and fertilization fails with disruption in any of these events. To examine differences in pollen tube growth in SI (stigmas pollinated by pollen from the same morphs, $\mathrm{P} \times \mathrm{P}$ and $\mathrm{T} \times \mathrm{T}$ ) and $\mathrm{SC}$ (stigmas pollinated by pollen from different morphs, $\mathrm{P} \times \mathrm{T}$ and $\mathrm{T} \times \mathrm{P}$ ) groups, the growth behaviors of pollen tubes of $P$. auriculata were observed by fluorescence microscopy (Figure 2). The pollen grain germinated at approximately $2 \mathrm{~h}$ (Figure $2 \mathrm{~h}$ ) only on SC stigmas; however, a longer period of time was required for germination of the SC pollen in the T group, and no pollen germination was observed at this timepoint for SI pollen. Pollen tubes in both pin and thrum flowers were elongated at approximately $4 \mathrm{~h}$ (Figure $2 \mathrm{j}$ and $\mathrm{I}$ ) and reached the ovary within $8 \mathrm{~h}$ (Figure $2 \mathrm{n}$ and $\mathrm{p}$ ). Conversely, no SI pollen stably adhered onto stigmas. According to the results, $2 \mathrm{~h}$ appears to be the ideal timepoint to detect differences between HetSI mechanisms.

\subsection{Pollen-Stigma Interactions in Compatible and Incompatible Groups}

The distinguishing characteristics between pollen grain and stigmas might be due to secondary characteristics in the SI process.

Environmental scanning electron microscopy (ESEM) was used to observe SC and SI pollen-stigma interactions of $P$. auriculata. For both morphs, each stigmatic lobe bears numerous multicellular structures that consist of a stalk and a head.

To ensure sufficient time for the pollen grain and stigmas to interact, pollen germination was examined approximately $2 \mathrm{~h}$ after artificial pollination. The "foot" structures penetrating the stigmas through papillae can be clearly observed in T×P pollinations (Figure 3c). Interestingly, P×T pollinations showed a distinct morphology; the stigmatic papillae around the germinated pollen became less globular (Figure 3a), and no "foot" structure was observed. In contrast, no pollen tubes and no shriveled stigmatic papillae were discovered in SI pollinations (Figure $3 \mathrm{~b}$ and d). To explore the relationship between metabolic 
changes and the mechanism of HetSI, UPLC-MS/MS was performed on extracts of different pollination combinations.

\subsection{Metabolite Identification by UPLC-MS/MS}

The microstructures showed that the SI pollen grain never germinated. However, in SC pollinations, the pollen grain germinated approximately $2 \mathrm{~h}$ after artificial pollination and successfully reached the ovaries within $8 \mathrm{~h}$. Thus, successful pollen adherence and hydration appear to be key points forsuccessful fertilization in P. auriculata, and samples for metabolite extraction were collected at $2 \mathrm{~h}$ after artificial pollination to assess differences between self-incompatibility and self-compatibility.

UPLC-MS/MS of the $P$. auriculata style metabolome revealed142 named metabolites identified or tentatively characterized in both positive and negative modes (Tables S1 and S2). The base peak intensity (BPI) chromatograms are shown in Figure 4. More specifically, PS (styles of the pin flowers), TS (styles of the thrum flowers), $\mathrm{P} \times \mathrm{P}, \mathrm{P} \times \mathrm{T}, \mathrm{T} \times \mathrm{T}$, and $\mathrm{T} \times \mathrm{P}$ samples mainly yielded amino acids, flavonoids, glycosides/sugars, phenols, amines, other organic acids, fatty acids/lipids and alcohols. A quality control (QC) sample was also generated to provide a set of data from which repeatability was assessed.

\subsection{Different Pollination Combinations Showed Distinct Metabolite Levels}

Principal component analysis (PCA) 2D plots of results from the 6 groups illustrated clear separation of the pollination samples into clusters according to their common spectral characteristics (Figure 5). This result means that the 6 groups can be easily differentiated.

Statistical analysis (OPLS-DA, FC analysis and T-test) was performed to calculate variations. In the

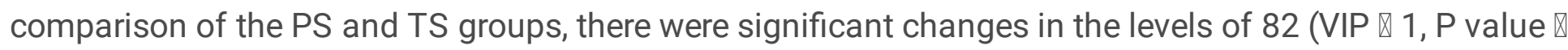
0.05) compounds. In the comparison of the $T \times T$ and $T \times P$ groups, there were significant changes in the levels of 66 (VIP $\otimes 1, P$ value $\otimes 0.05$ ) compounds, and in the comparison of the P×T and P P groups, there were significant changes in the contents of 66 metabolites.

To systematically evaluate biomarkers, a heatmap was generated (Figure 6), which revealed marked variation in metabolite abundance for the different pollinations. Correspondingly, an obvious separation could be observed between styles without pollinations ( $P S, T S)$ and pollinated styles ( $P \times P, P \times T, T \times P, T \times T)$. In Figure 6a, PS and TS were grouped into 2 classes. In Figure $6 \mathrm{~b}$ the 32 samples were clearly grouped into 2 classes: 16 samples for SC and SI styles in the P group and 16 samples for SC and SI styles in the $T$ group Furthermore, 4 subclasses consisted of samples from styles in different pollinations $(P \times P, P \times T$, $T \times P, T \times T)$.

\subsubsection{Amino Acids}


Twenty-one types of amino acids were detected, and their levels were compared between the PS and TS groups. The total content of amino acids was higher in TS (Figure 7a). Fourteen types of amino acids showed significant changes (VIP $\otimes 1, P$ value $\otimes 0.05$ ) in relative content. Glutamine, glutathione, glutamic acid, isoleucine, $\mathrm{N}$-acetyl-L-phenylalanine, prolyl-tryptophan, aspartic acid, pipecolic acid, lysine and Nacetyl-L-aspartic acid showed distinct increases in TS, whereas only glutathione disulfide, tyrosine, isoleucyl-proline and leucyl-phenylalanine were higher in PS.

Compared with the levels in T×P, 8 types of amino acids showed distinct increases in T×T: anthranilic acid (Vitamin L1), glutathione, phenylalanine, glutamine, tyrosine, leucyl-phenylalanine, pipecolic acid and $\mathrm{N}$-acetyl-L-aspartic acid. Unsurprisingly, a similar trend in total amino acid contents was observed for PxT and $\mathrm{P} \times \mathrm{P}$ (Figure 7a), including glutathione, arginine, histidine, isoleucine, $\mathrm{N}$-acetyl-L-aspartic acid, $\mathrm{N}$ acetyl-L-phenylalanine, tryptophan, isoleucyl-proline, leucyl-phenylalanine, leucyl-leucine, pipecolic acid and lysine.

\subsubsection{Flavonoids}

Compare with PS, the total content of flavonoids showed distinct increases in TS (Figure 7b). Myricetin, myricitrin, and procyanidin B2 changed significantly and were more abundant in TS. However, 3-0methylquercetin, kaempferol, kaempferol-3-0-rutinoside, rutin, quercitrin, quercetin, isorhamnetin and isoquercitin decreased sharply.

In T×T and T×P, flavonoids levels changed significantly. Compared with SC pollinations, myricetin increased in SI pollinations, whereas taxifolin, 3-o-methylquercetin, kaempferol, kaempferol-3-o-rutinoside, isorhamnetin, procyanidin b2 and rutin decreased sharply. In the pin-female parent group, 3-omethylquercetin and isorhamnetin increased in the SI group, but kaempferol, kaempferol-3-o-rutinoside, myricetin, quercetin, procyanidin b2, myricitrin and rutin remained low.

\subsubsection{Glycosides/Sugars}

The levels of glycosides/sugars remained higher in TS (Figure 7c), and 5 types of glycosides/sugars showed significant changes (VIP $\otimes 1, P$ value $\otimes 0.05$ ) when the PS and TS groups were compared. Adenosine monophosphate (AMP) and glucoronate were increased in TS, whereas guanosine, inosine and S-methyl-5'-thioadenosine decreased.

The content of glycosides/sugars in SC and SI included adenosine, glucuronate, mannose, trehalose, guanosine, inosine, alpha-hederin, xanthosine, maltose, sucrose and S-methyl-5'-thioadenosine. The total content was much higher in the SC group than in the SI group (Figure 7c).

\subsubsection{Other Organic Acids}

A total of 33 other organic acids were detected. Unsurprisingly, compared with SP, other organic acids remained at higher levels in TS, and 17 showed significant changes (VIP $\otimes 1, P$ value $\otimes 0.05)$. TCA intermediates such as citrate and malic acid were all abundant in TS (Figure 7c). 
Intermediates of TCA were included in other organic acids. In T×T and T×P, intermediates of TCA were much higher in the SI group than in the SC group. Unsurprisingly, the same trend was found in $\mathrm{P} \times \mathrm{P}$ and $\mathrm{P} \times \mathrm{T}$ (Figure 7i).

\subsubsection{Fatty Acids/Lipids}

Thirty named fatty acids/lipids were detected, including 16 that showed significant changes. Cutin, suberin and wax biosynthesis of 16-hydroxypalmitic acid remained higher in TS. Glyoxylate and dicarboxylate metabolism of tartaric acid and meso-tartaric acid were also abundant in TS. However, fatty acid biosynthesis of linoleic acid decreased in TS compared with PS.

After SI pollination, the total content of fatty acids decreased in the T group, and the corresponding content in the $\mathrm{P}$ group showed the same trend (Figure 7e).

\subsubsection{Alcohols}

Eight types of alcohols were detected. Among them, phytosphingosine, uvaol, 1-oleoyl-sn-glycerol 3phosphate, 1-stearoyl-sn-glycerol 3-phosphocholine and ascorbic acid showed significant changes. The content of phytosphingosine increased in TS, and ascorbic acid and glutathione metabolism showed the same trend in TS.

The alcohol content showed an opposite tendency in the T and P groups, with higher levels in SI pollinations than in SC pollinations in the T group but lower levels in SI pollinations in the P group (Figure $7 f)$.

\subsubsection{Amines}

Amines were more abundant in TS than PS. Four types of amines changed significantly. Betaine, one of the metabolites found in glycine, serine and threonine metabolism, showed distinct increases in TS, and glycerophosphocholine showed the same trend. Spermidine and phenylethylamine changed in an opposite manner.

The total content of amines in SI pollinations was reduced compared to that in SC pollinations in the P groups (Figure 7h) and remained steady in the T groups. 3-Methoxytyramine, phytosphingosine, spermidine, tryptamine and tyramine were significantly altered in $\mathrm{T} \times \mathrm{T}$ and $\mathrm{T} \times \mathrm{P}$. Four types of amines showed obvious changes in $\mathrm{P} \times \mathrm{T}$ and $\mathrm{P} \times \mathrm{P}$.

\subsubsection{Other Metabolites}

Other metabolites included phenols, aldehydes, alkaloids, steroids and heterocyclic compounds. Phenols were richer in TS than in PS. Indole (heterocyclic compounds) and 3-formylindole (heterocyclic compounds) remained higher in TS, whereas caffeine (alkaloids), 3-methyluric acid (alkaloids) and theobromine (steroids) showed the opposite trend. 
Some aldehydes such as phenylacetaldehyde decreased significantly in the T group after SI pollination, although vanillin increased. Compared with SC pollinations, an increase in uric acid (alkaloids) was observed in SI pollinations, whereas a higher level of 3-formylindole (heterocyclic compounds) was found in SI pollinations. In addition, biliverdin (porphyrin) remained richer in SI pollinations. The content of phenols was much higher in SC pollinations than in SI pollinations, and only resveratrol significantly changed and remained more abundant in SI pollinations. In the P group, vanillin content decreased after SI pollination. Alkaloid was more abundant in SI pollinations. Phenols increased after SI pollination, and phloretin decreased after SI pollination; however, resveratrol showed the opposite pattern.

\section{Discussion}

In this study, 142 named metabolites were identified in 2 morphs of styles and 4 different pollinations. According to PCA, PS, TS, T XT, T×P, P $\times T$ and P×P were separated from each other, indicating that metabolites from the $\mathrm{T}$ group and the $\mathrm{P}$ group were clearly distinguished. $P$. auriculata showed distinct metabolic changes for self-incompatibility in pin and thrum flowers.

According to the results of fluorescence, pollen tube growth showed a delay in the P group. The mature style stores a large amount of nutrients for pollen tube growth. Moreover, the levels of TCA intermediates (citrate, isocitrate, malic acid, and cis-aconitate), amino acids, phenols, and flavonoids remained higher in TS, which may support faster pollen tube growth. In addition, ESEM showed the microstructure of pollenstigma interactions. No liquid was present on the stigma surface, precluding adhesion; thus, a dry stigma is typical. Overall, rich fatty acids and lipids in SC pollination in both the P and T groups play an important role in the hydrophobic nature of pollen-stigma capture and pollen tube growth [19-21].

Cell wall loosening in the stigma is important for pollen tube growth [22] and flavonoids are essential for pollen germination and tube growth and may be involved in cell wall loosening, thus facilitating penetration of the SC stigma by the pollen tube and consequently the supply of flavonoids by either the pollen or stigma at pollination [23-28]. For instance, kaempferol increased in SC samples when pollen tubes grew into the pistil-transmitting tissue. This result is consistent with previously reported results for petunia $[29,30]$.

Pollen tube growth is a highly energy-consuming process involving many related pathways. After successful recognition, high-energy nutrients must be metabolized to produce acetyl-CoA, which is incorporated into the pollen tube's TCA cycle, ultimately enhancing ATP production to promote pollen tube growth [31]. The superpathway is composed of many metabolites that construct a style metabolic network with annotated pathway information in KEGG. After germination, the pollen tube grows into the extracellular matrix in the stigma secretory zone, and pistil transmitting tract cells secrete amino acids, soluble sugar (sucrose) and phenols into the extracellular matrix, providing nutrients and resources that support rapid pollen germination and pollen tube growth after the materials stored within the pollen grain are exhausted [32, 33]. Moreover, the TCA intermediates (citrate, isocitrate, malic acid, and cis-aconitate) and amino acids remained higher in SI pollination than in SC pollination in both the T and $\mathrm{P}$ groups. 
Without pollen growth, metabolites remain at a relatively high level. Zhao asserted that tomato SI might be due to lack of high-energy nutrients for pollen growth. However, the results of our research indicate that HetSI might not be related to a lack of high-energy nutrients to facilitate pollen tube growth, which is completely different from Zhao's hypothesis regarding the self-incompatibility mechanism of tomatoes. Moreover, Zechmann asserted that glutathione is essential for pollen germinations, however, glutathione showed a higher level in SI pollinations which means glutathione deficiency may not the reason for SI [37].

Surprisingly, the total contents of flavonoids, amines and alcohols changed in an opposite way in the $\mathrm{P}$ and T groups: the content was higher in SI samples than in SC samples in the T group, but the opposite was found in the $\mathrm{P}$ group. This result might be due to the extremely complex mechanism and growth rate variation of pollen tubes. Genetic and genomic approaches have shown that initial pollen tube growth is autotrophic and utilizes the nutrients stored in pollen grains; for subsequent growth, heterotrophic pollen tubes absorb and metabolize external energy-rich metabolites from the stigma for energy generation (ATP) [32, 34, 35]. After rehydration, metabolites in pollen are quickly reformed due to enzyme reactivation [36]. According to our fluorescence microscopy results, pollen germination was delayed in $\mathrm{P} \times \mathrm{T}$ pollinations, and no pollen tube was observed, which may result in metabolic differences due to the variation in pollen tube growth capacity. Compared with the metabolites in the desiccated pollen in the SI group of the P group, the metabolites in rehydrated pollen in the SC group might have accumulated to support the initial autotrophic stage of pollen tube growth. On the other hand, growing pollen tubes may also consume high-energy nutrients from the style [31], including flavonoids, amines, fatty acids/lipids and alcohols. Thus, the sharp decline in SC pollinations in the T group might be due to the consumption of supplements for pollen tube growth [33].

\section{Conclusions}

In this research, we investigated the morphological and metabolic differences in different types of styles and SC and SI pollinations of Plumbago auriculata. Metabolic discrimination between SC and SI during pollen tube growth by UPLC-MS/MS in HetSI plants has not been reported previously. Moreover, 8 biological replicates of metabolic analysis were carried out with high coincidence and good repeatability. A total of 142 chemical compounds were identified. The metabolites were primarily distributed over 8 chemical families, including amino acids, flavonoids, phenols, glycosides/sugars, other organic acids, fatty acids/lipids, amines and alcohols. The metabolomes of $P S$, TS, $P \times P, P \times T, T \times T$, and $T \times P$ pollinations exhibited distinct metabolic changes. There was an abundance of amino acids, flavonoids, phenols, alcohols and tricarboxylic acid cycle-related metabolites in SI pollinations, suggesting that the lack of high-energy nutrients might not be the source of self-incompatibility. The growth rate differences of the pollen tube might also result in metabolic differences in PS and TS. These results provide supplementary data for HetSI as well as a basis for prospective works.

\section{Methods}




\subsection{Materials and Reagents}

Floral styles were collected from samples of Plumbago auriculata pollinated in different manners (PS, TS, $\mathrm{P} \times \mathrm{P}, \mathrm{P} \times \mathrm{T}, \mathrm{T} \times \mathrm{T}, \mathrm{T} \times \mathrm{P})$. The plants were grown in a greenhouse at Sichan Agricultural University for generations to exclude pollinators and to prevent predation. Styles for morphological study were pollinated at 8:00 AM and collected after 1, 2, 4, and $8 \mathrm{~h}$. Pin and thrum styles were collected without pollinations (50 styles for each sample, a total of 400 styles were prepared for each type). Fifty pollinated styles after 1, 2, 4, and $8 \mathrm{~h}$ were prepared for each pollination $(P \times P, P \times T, T \times T, T \times P)$. A total of 400 (approximately $400 \mathrm{mg}$ ) pollinated styles were prepared for each pollination ( $P \times P, P \times T, T \times T$, and $T \times P$ ) after 2 h.Styles for metabolome analysis were pollinated at 8:00 AM and collected after $2 \mathrm{~h}(10: 00 \mathrm{AM})$.

\subsection{Staining and Microscopy}

\subsubsection{Fluorescence Microscopy}

Floral styles at 1, 2, 4, and $8 \mathrm{~h}$ after pollination were collected and fixed in ethanol:acetic acid (2:1, v:v) for $12 \mathrm{~h}$. The fixed stigmas were cleared with $8 \mathrm{~mol} / \mathrm{L} \mathrm{NaOH}$ for $6 \mathrm{~h}$, rinsed thoroughly in distilled water, washed three times with $\mathrm{K}_{3} \mathrm{PO}_{4}(0.1 \mathrm{~mol} / \mathrm{L})$, soaked for $20 \mathrm{~min}$ and stained with $0.05 \%$ achromatic aniline blue. Pollen grain and stigma interactions were detected by fluorescence microscopy. Each experiment was replicated 30 times.

\subsubsection{Environmental Scanning Electron Microscopy}

Fresh samples of styles from $P \times P, P \times T, T \times T, T \times P$ pollinations were directly adhered onto stages using conducting resin and placed in a special low-pressure chamber of a Quanta $250 \mathrm{FEl}$ microscope. Samples were observed in environmental scanning mode. For observation, the pressure was set to 150 $\mathrm{Pa}$, and the tension was set to $500 \mathrm{kV}$. Micrographs were interpreted automatically using the hardware and software supplied with the microscope. Each experiment was replicated five times.

\subsection{Sample Preparation and Extraction}

Two-hour-pollinated styles were randomly collected from different pollination combinations (50 styles for each sample) and immediately frozen in liquid nitrogen and stored at $-80^{\circ} \mathrm{C}$ for metabolite extraction. Eight biological replicates were prepared for this research. All samples were ground into homogeneous powder (50 mg for each sample), which was then extracted with $1 \mathrm{ml}$ of methanol:acetonitrile:water $(2: 2: 1, v: v: v)$ to form a turbid suspension that was ultrasonically treated twice for 30 min at ultralow temperature. The sample was incubated for $1 \mathrm{~h}$ at $-20^{\circ} \mathrm{C}$ for protein precipitation. After centrifugation at $13,000 \mathrm{rpm}$ for $15 \mathrm{~min}$ at $4^{\circ} \mathrm{C}$, the supernatant was completely removed using a vacuum concentrator. The sample was redissolved in $100 \mu \mathrm{l}$ of acetonitrile:water (1:1, v:v) and centrifuged at $14,000 \mathrm{xg}$ for 15 min at $4^{\circ} \mathrm{C}$. The supernatant was injected directly into the UPLC system. The volume injected for each run was $10 \mu \mathrm{l}$. A quality control (QC) sample was prepared by mixing aliquots from each of the samples. 


\subsection{UPLC-MS/MS}

UPLC analysis was performed using an Agilent 1290 Infinity LC UPLC system with the HILIC column. The mobile phases consisted of eluents A (water $+25 \mathrm{mM}$ ammonium acetate $+25 \mathrm{mM}$ ammonia) and $\mathrm{B}$ (acetonitrile), with a flow rate of $0.3 \mathrm{ml} / \mathrm{min}$ and a linear gradient program as follows: $95 \% \mathrm{~B}$ from 0 to 0.5 $\min , 95 \%-65 \%$ B from 0.5 to $7 \mathrm{~min}, 65 \%$ - $40 \%$ B from 7 to $9 \mathrm{~min}, \mathrm{~B}$ maintained at $40 \%$ from 9 to $10 \mathrm{~min}$, $40 \%-95 \%$ B from 10 to $11.1 \mathrm{~min}$, and $B$ remaining at $95 \%$ from 11.1 to $16 \mathrm{~min}$. The temperature of the UPLC column and autosampler were set at $25^{\circ} \mathrm{C}$ and $4^{\circ} \mathrm{C}$, respectively.

The MS experiments were performed using a Triple-TOF 5600 mass spectrometer (AB SCIEX) connected to the UPLC system through an electrospray ionization (ESI) interface. The optimized instrumental parameters were as follows: ion source gas1 (Gas1), 60; ion source gas2 (Gas2), 60; curtain gas (CUR), 30; source temperature, $600^{\circ} \mathrm{C}$; ion spray voltage floating (ISVF), $\pm 5500 \mathrm{~V}$ (positive/negative mode); TOF MS scan $\mathrm{m} / \mathrm{z}$ range, 60-1200 Da; product ion scan $\mathrm{m} / \mathrm{z}$ range, 25-1200 Da; TOF MS scan accumulation time, $0.15 \mathrm{~s} / \mathrm{spectrum}$; and product ion scan accumulation time, $0.03 \mathrm{~s} / \mathrm{spectrum}$. Secondary mass spectrometry data were acquired using information dependent acquisition (IDA) in high-sensitivity mode with declustering potential (DP) of $\pm 60 \mathrm{~V}$ (positive/negative mode), collision energy of $30 \mathrm{eV}$; IDA excluded isotopes within $4 \mathrm{Da}$, and 6 candidate ions were monitored per cycle. The QCs were injected at regular intervals (every 8 samples) throughout the analytical run to provide a set of data.

\subsection{Data Analysis}

For the screening analysis, raw data were transferred to .mzXML files using ProteoWizard. The XCMS program was applied for assessing peak alignments, retention time corrections, and peak area extraction. A tolerance of mass accuracy ( $<25 \mathrm{ppm}$ ) and second-order spectrum matching were applied to identify the chemical structures of metabolites. The resulting data were analyzed by PCA, orthogonal projections to latent structures discriminant analysis (OPLS-DA), partial least squares discriminant analysis (PLSDA), fold change analysis and the t-test. Information for chemical compounds were obtained from mass spectrum, retention times, a self-built database and METLIN. Metabolites were compared by peak areas. Heatmaps were generated in R-language. Eight biological replicates were treated as an average per sample for subsequent metabolite analysis.

\section{List Of Abbreviations}

Sl: self-incompatibility

SC: self-compatibility

TS: the styles of thrum flowers

PS: the styles of pin flowers 
P×P: pin-morph stigmas pollinated with pin pollens

$\mathrm{P} \times \mathrm{T}$ : pin-morph stigmas pollinated with thrum pollens

$\mathrm{T} \times \mathrm{T}$ : thrum -morph stigmas pollinated with thrum pollens

T×P: thrum -morph stigmas pollinated with pin pollens

The T group: PS, $P \times P, P \times T$

The P group: TS, $T \times T, T \times P$

UPLC-MS/MS: ultra performance liquid chromatography-coupled mass spectrometry

PCA: principal component analysis

TCA: tricarboxylic acid cycle

QC: quality control

ANOVA: analysis of variance

\section{Declarations}

\section{Supplementary Materials:}

Table S1. xlsx

Additional file 1: Relative content of all the metabolites in PS, TS, P×P, P×T, T×T, T×P and QC. (EXCEL 133KB)

Table S2. xlsx

Additional file 2: Relative content of metabolites in PS and TS. Table S2 represent significant changes in

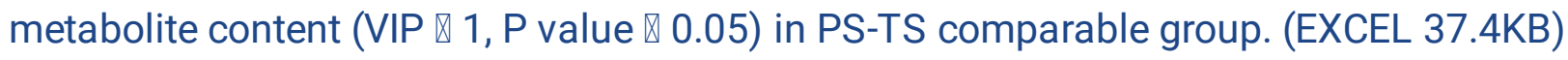

Table S3. xlsx

Additional file 3: Relative content of metabolites in T×T and T×P. Table S3 represent significant changes in metabolite content (VIP $\otimes 1, P$ value $\otimes 0.05$ ) in $T \times T-T \times P$ comparable group. (EXCEL 32.7KB)

Table S4. xlsx

Additional file 4: Relative content of metabolites in PxP and P×T. Table S4 represent significant changes in metabolite content (VIP $\otimes 1, P$ value $\otimes 0.05$ ) in $P \times P$ - $P \times T$ comparable group. (EXCEL 33.1KB) 
Not applicable

\section{Consent for Publication}

Not applicable

\section{Availability of Data and Material}

All data generated or analysed during this study are included in this published article [and its supplementary information files].

\section{Competing Interests}

The authors declare that they have no conflicts of interest concerning this article.

\section{Funding}

National Key R\&D Program of China, (2018YFD0600105)

Sichuan Science and Technology Program, (2018JY0211).

\section{Author Contributions}

Experimental design, Suping Gao and Ting Lei. Experiment and result analysis, Di Hu and Wenji Li.

Experimental verification, Ju Hu. Paper writing, Di Hu and Wenji Li. Tabulation, Ping Shen and Yurong Li. Revision, Jiani Li.

\section{Acknowledgements}

The study was supported by the National Key R\&D Program of China, (2018YFD0600105), Sichuan Science and Technology Program, (2018JY0211).

\section{References}

1. Darwin C. On the various contrivances by which British and foreign orchids are fertilised by insects: and on the good effects of intercrossing. London: John Murray; 1862.

2. Ganders FR. Disassortative pollination in the distylous plant Jepsonia heterandra. Can J Bot. $1974 ; 52: 2401-6$.

3. Kohn JR, Barrett SCH. Experimental studies on the functional significance of heterostyly. Evolution. 1992;46:43-55.

4. Lloyd DG, Webb CJ. The evolution of heterostyly. In: Barrett, SCH, editor. Evolution and function of heterostyly. Berlin: Springer; 1992. p. 151-78.

5. Barrett SC. Evolution and function of heterostyly. Berlin: Springer-Verlag; 1992. 
6. Barrett SCH, Cruzan MB. Incompatibility in heterostylous plants. In: Williams EG, Clarke AE, Knox RB, editors. Genetic control of self-incompatibility and reproductive development in flowering plants advances in cellular and molecular biology of plants. Dordrecht: Springer; 1994. p. 189-219.

7. Tugizimana F, Piater L, Dubery I. Plant metabolomics: a new frontier in phytochemical analysis. S Afr J Sci. 2012;109:1-11.

8. Fernie AR. The future of metabolic phytochemistry: larger numbers of metabolites, higher resolution, greater understanding. Phytochemistry. 2008;68:2861-80.

9. Verpoorte R, Choi YH, Kim HK. NMR-based metabolomics at work in phytochemistry. Phytochem Rev. 2007;6:3-14.

10. Fiehn O. Metabolomics - the link between genotypes and phenotypes. Plant Mol Biol. 2002;48:15571.

11. Kim HK, Choi YH, Verpoorte R. NMR-based plant metabolomics: where do we stand, where do we go? Trends Biotechnol. 2011;29:267-75.

12. Yu C, Luo X, Zhan X, Hao J, Zhang L, Song YB, Shen CJ, Dong M. Comparative metabolomics reveals the metabolic variations between two endangered Taxus species (T. fuana and T. yunnanensis) in the Himalayas. BMC Plant Biology, 2018, 18:197.

13. Faehnrich B, Kraxner C, Kummer S, Franz C. Pollen tube growth and self incompatibility in Matricaria recutita. Euphytica. 2015;206:357-63.

14. Samuel MA, Chaal BK, Lampard G, Green BR, Ellis BE. Surviving the passage: non-canonical stromal targeting of an Arabidopsis mitogen-activated protein kinase. Plant Signal Behav. 2008;3:6-12.

15. Samuel MA, Mudgil Y, Salt JN, Delmas F, Ramachandran S, Chilelli A, et al. Interactions between the Sdomain receptor kinases and AtPUB-ARM E3 ubiquitin ligases suggest a conserved signaling pathway in Arabidopsis. Plant Physiol. 2008;147:2084-95.

16. Haasen KE, Goring DR. The recognition and rejection of self-incompatible pollen in the Brassicaceae. Bot Stud. 2010;51:1-6.

17. Zhao P, Pan Q, Yu W, Zhao L. Dissect style response to pollination using metabolite profiling in selfcompatible and self-incompatible tomato species. J Chromatogr B. 2016;1017-1018:153-62.

18. Ferrero V, de Vega C, Stafford GI, Van Staden J, Johnson SD. Heterostyly and pollinators in Plumbago auriculata (Plumbaginaceae). S Afr J Bot. 2009;75:778-84.

19. Heizmann P, Luu DT, Dumas C. Pollen-stigma adhesion in the Brassicaceae. Ann Bot. 2000;85:23-7. 
20. Fragallah S, Wang P, Li N, Chen Y, Lin S. Metabolomic analysis of pollen grains with different germination abilities from two clones of Chinese fir (Cunninghamia lanceolata (Lamb) Hook). Molecules. 2018;23:E3162.

21. Tan H, Zhang J, Qi X, Ye W, Wang X, Xiang X. Integrated metabolite profiling and transcriptome analysis reveals a dynamic metabolic exchange between pollen tubes and the style during fertilization of Brassica napus. Plant Mol Biol. 2018;97:325-35.

22. Elleman CJ, Franklin-Tong V, Dickinson HG. Pollination in species with dry stigmas: the nature of the early stigmatic response and the pathway taken by pollen tubes. New Phytol. 1992;121:413-24.

23. Mo Y, Nagel C, Taylor LP. Biochemical complementation of chalcone synthase mutants defines a role forflavonoids in functional pollen. Proc Natl Acad Sci USA. 1992;89:7213-7.

24. Taylor LP, Jorgensen R. Conditional male fertility in chalcone synthase-deficient petunia. J Hered. 1992;83:11-7.

25. Ylstra B, Busscher J, Franken J, Hollman PCH, Mol JNM, van Tunen AJ. Flavonols and fertilization in Petunia hybrida: localization and mode of action during pollen tube growth. Plant J. 1994;6:201-12.

26. Vogt T, Wollenweber E, Taylor LP. The structural requirements of flavonols that induce pollen germination of conditionally male fertile petunia. Phytochemistry. 1995;38:589-92.

27. Xu P, Vogt T, Taylor LP. Uptake and metabolism of flavonols during in-vitro germination of Petunia hybrida (L.) pollen. Planta. 1996;202:257-65.

28. Miller KD, Guyon V, Evans JNS, Shuttleworth WA, Taylor LP. Purification, cloning, and heterologous expression of a catalytically efficient flavonol 3-0-Galactosyltransferase expressed in the male gametophyte of Petunia hybrida. J Biol Chem. 1999;274:34011-9.

29. Veit M, Pauli GF. Major flavonoids from Arabidopsis thaliana leaves. J Nat Prod. 1999;62:1301-3.

30. Kerhoas L, Aouak D, Cingöz A, Routaboul J-M, Lepiniec L, Einhorn J, et al. Structural characterization of the major flavonoid glycosides from Arabidopsis thaliana seeds. J Agric Food Chem. 2006;54:6603-12.

31. Yue X, Gao XQ, Wang F, Dong Y, Li X, Zhang XS. Transcriptional evidence for inferred pattern of pollen tube-stigma metabolic coupling during pollination. PLoS One. 2014;9:e107046.

32. Labarca $C$, Loewus $F$. The nutritional role of pistil exudate in pollen tube wall formation in Lilium longiflorum: II. Production and utilization of exudate from stigma and stylar canal. Plant Physiol. 1973;52:87-92.

33. Goetz M, Guivarch A, Hirsche J, Bauerfeind MA, Gonzalez MC, Hyun TK, et al. Metabolic control of tobacco pollination by sugars and invertases. Plant Physiol. 2017;173:984-97. 
34. O'Kelley JC. External carbohydrates in growth and respiration of pollen tubes in vitro. Am J Bot. 1955;42:322-7.

35. Obermeyer G, Fragner L, Lang V, Weckwerth W. Dynamic adaption of metabolic pathways during germination and growth of lily pollen tubes after inhibition of the electron transport chain. Plant Physiol. 2013;162:1822-33.

36. Fíla J, Radau S, Matros A, Hartmann A, Scholz U, Feciková J, et al. Phosphoproteomics profiling of tobacco mature pollen and pollen activated in vitro. Mol Cell Proteom. 2016;15:1338-50.

37. Zechmann B, Koffler BE, Russell SD. Glutathione synthesis is essential for pollen germination in vitro. BMC Plant Biology, 2011, 13:173.

\section{Figures}

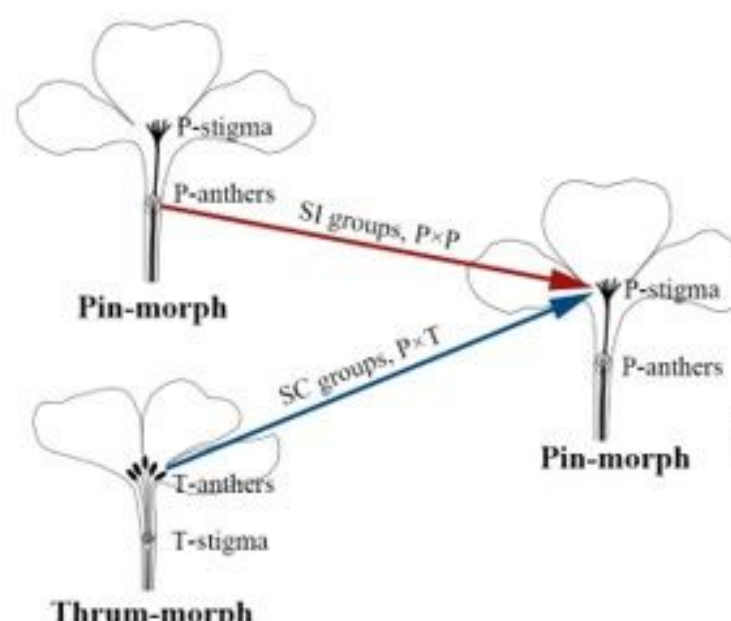

Thrum-morph

P-group

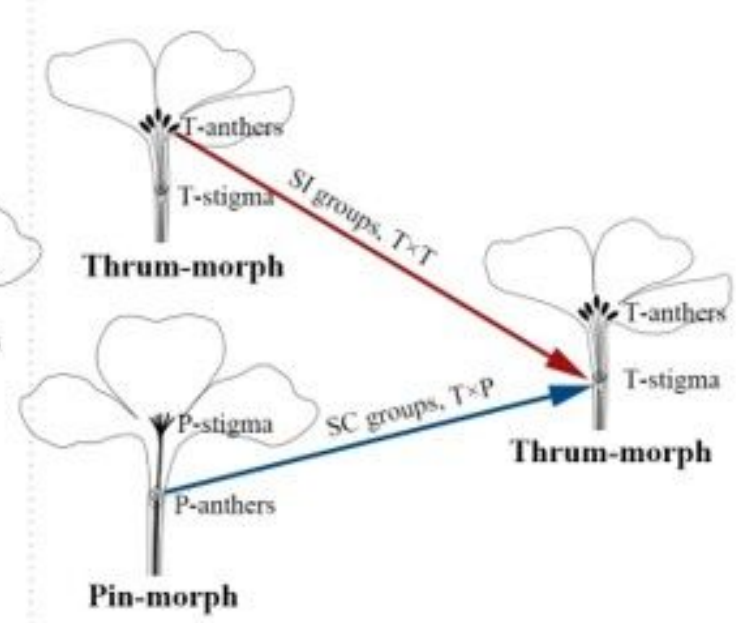

T-group

\section{Figure 1}

Pin-morph flower $(P)$ and thrum-morph flower $(T)$. The P group (PS: the styles of pin flowers; PxP: pinmorph stigmas pollinated with pin pollens; $\mathrm{P} \times \mathrm{T}$ : pin-morph stigmas pollinated with thrum pollens). The T group (TS: the styles of thrum flowers; $T \times T$ : thrum-morph stigmas pollinated with thrum pollens; $T \times P$ : thrum-morph stigmas pollinated with pin pollens). The red arrows indicate self-incompatible (SI) pollinations and the blue arrows self-compatible (SC) pollinations. 

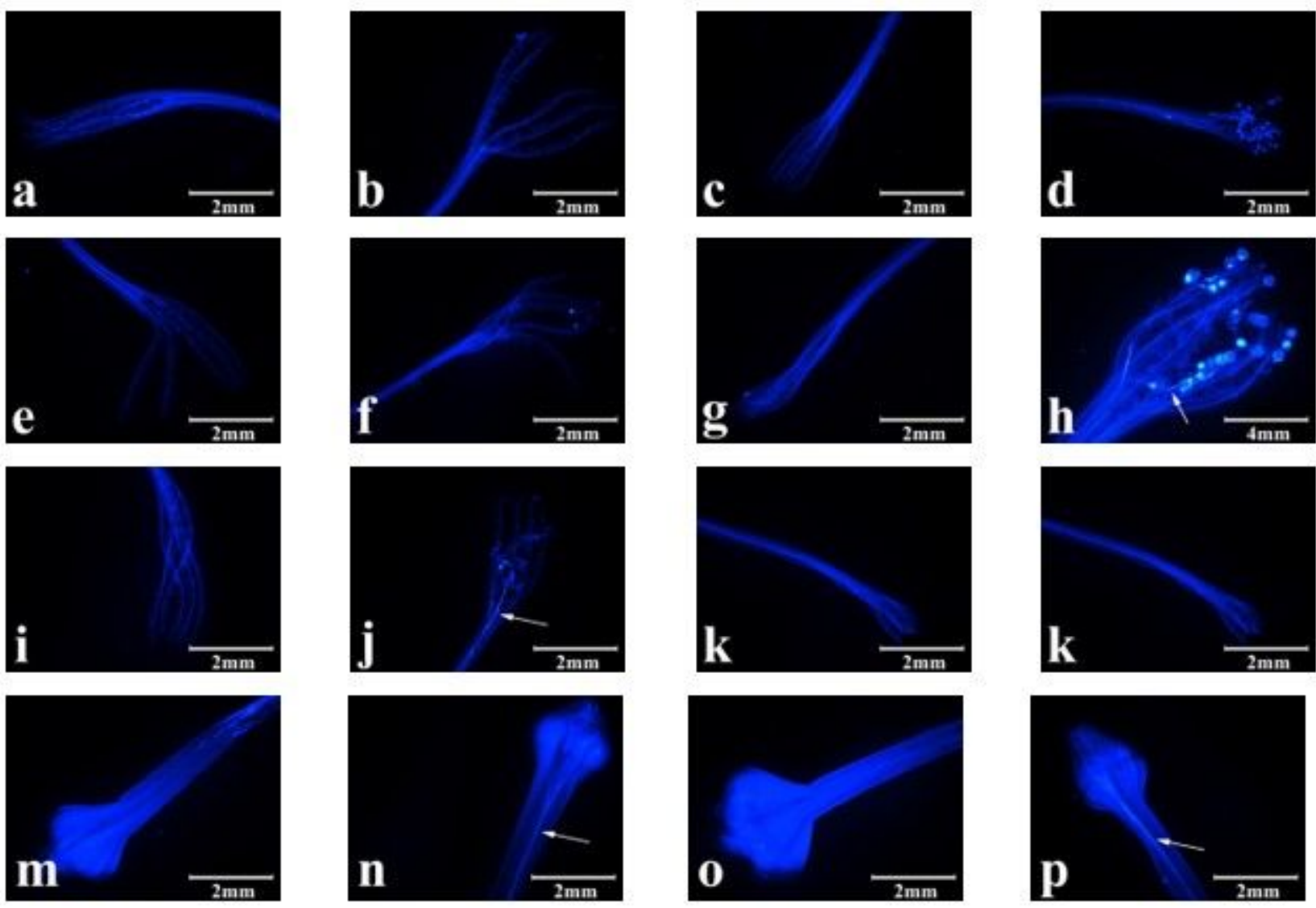

\section{Figure 2}

The development of pollen tubes after $1,2,4$, and $8 \mathrm{~h}$ in SI and SC pollination. (a, e, i, m) PxP pollinations, $1 \mathrm{~h}, 2 \mathrm{~h}, 4 \mathrm{~h}$, and $8 \mathrm{~h}$ from top to bottom; (b, f, j, n) PxT pollinations, $1 \mathrm{~h}, 2 \mathrm{~h}, 4 \mathrm{~h}$, and $8 \mathrm{~h}$ from top to bottom; (c, g, k, o) T×T pollinations, $1 \mathrm{~h}, 2 \mathrm{~h}, 4 \mathrm{~h}$, and $8 \mathrm{~h}$ from top to bottom; (d, h, l, p) T×P pollinations, 1 $\mathrm{h}, 2 \mathrm{~h}, 4 \mathrm{~h}$, and $8 \mathrm{~h}$ from top to bottom. The direction of the white arrows indicates the position of the pollen tube. 

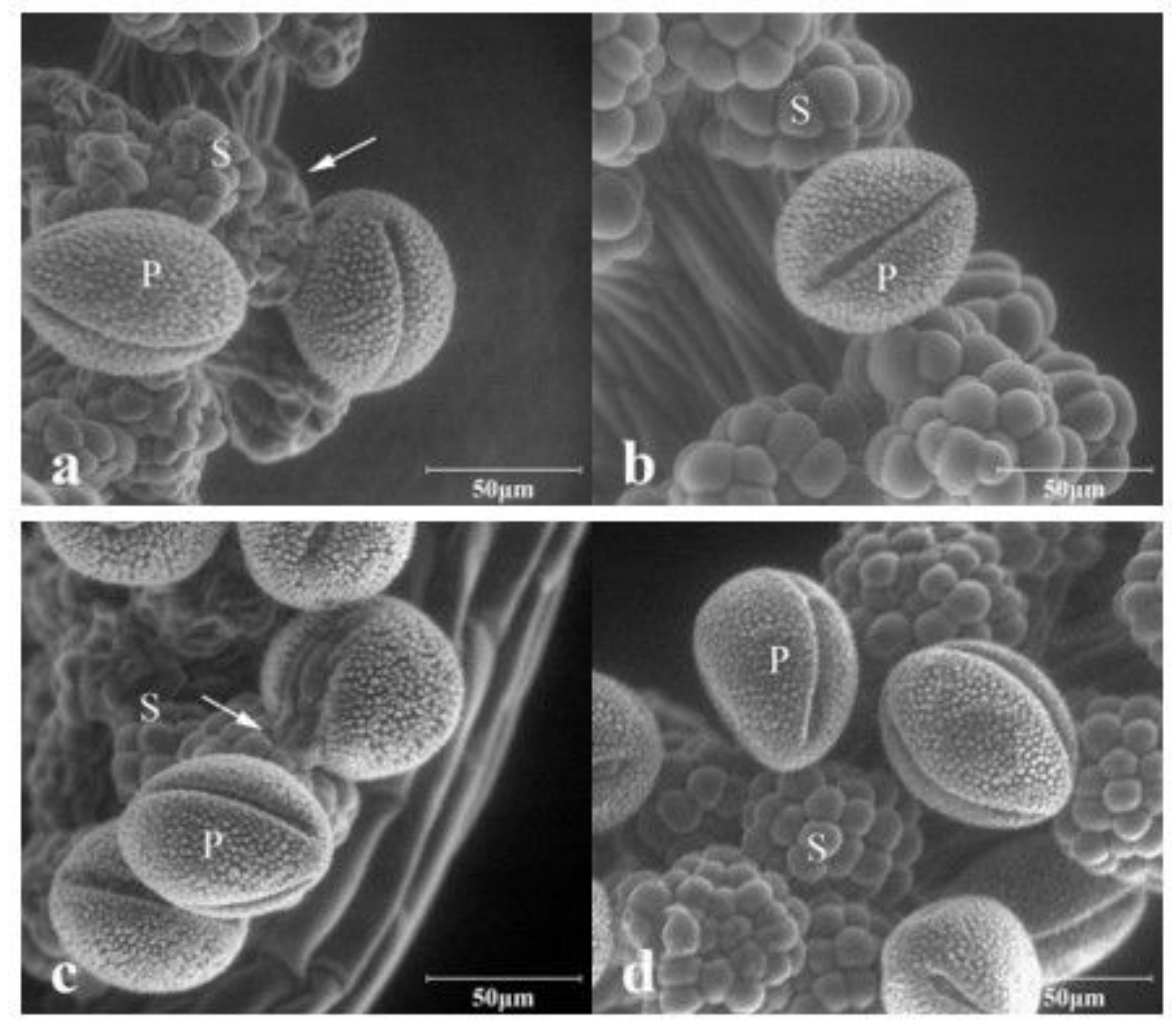

\section{Figure 3}

Pollen-stigma interactions after different pollinations for 2 h. (a) PxT; (b) P×P; (c) T×P; (d) T×T. The direction of the white arrows indicates the position of the pollen tube. P: pollen; S: stigma. 

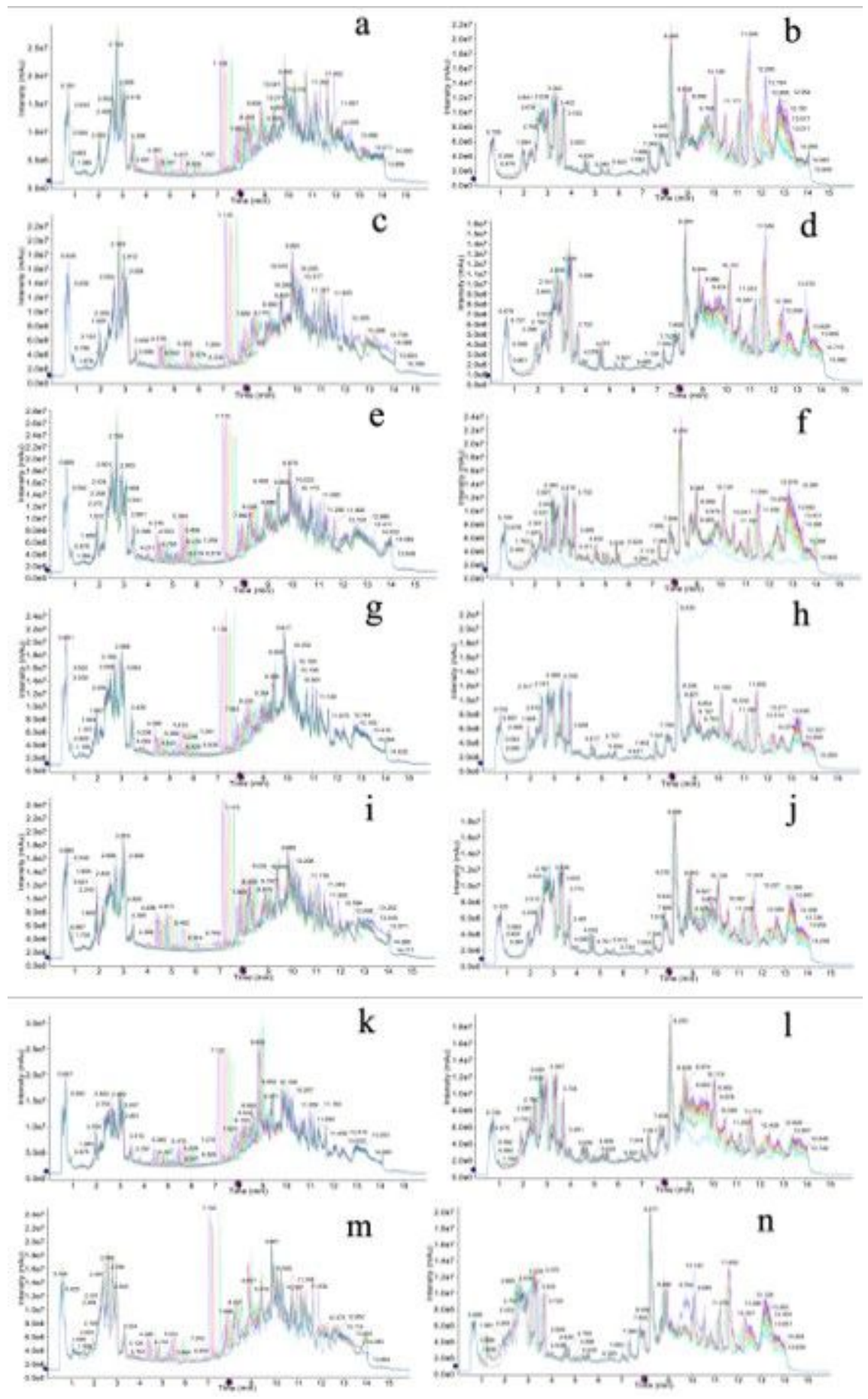

\section{Figure 4}

Total ion chromatograms from different pollinations (positive and negative modes). (a) PS positive mode; (b) PS negative mode; (c) TS positive mode; (d) TS negative mode; (e) PXP positive mode; (f) P×P negative mode; (g) PxT positive mode; (h) P×T negative mode; (i) T×T positive mode; (j) $T \times T$ negative mode; (k) T×P positive mode; (I) T×P negative mode, (m) QC positive mode; ( $\mathrm{n}$ ) QC negative mode. 

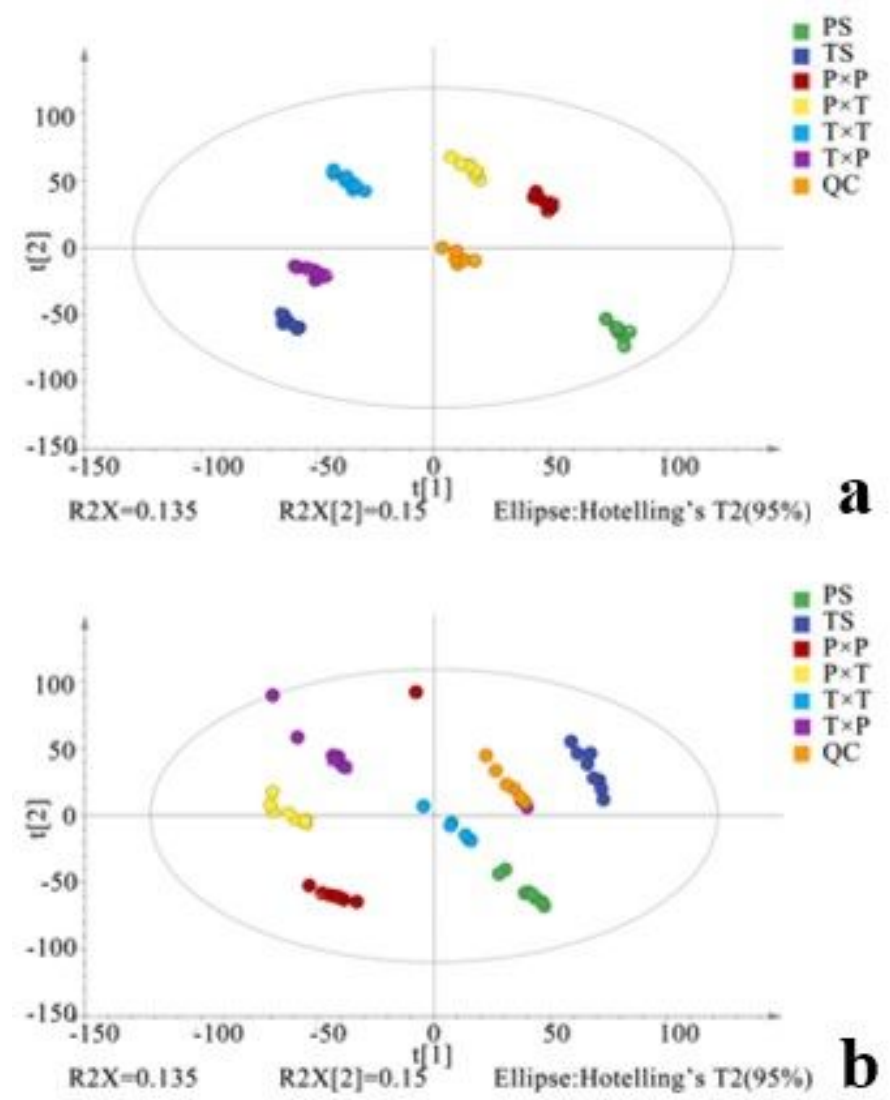

Figure 5

PCA of different pollinations in positive mode (a) and negative mode (b). 

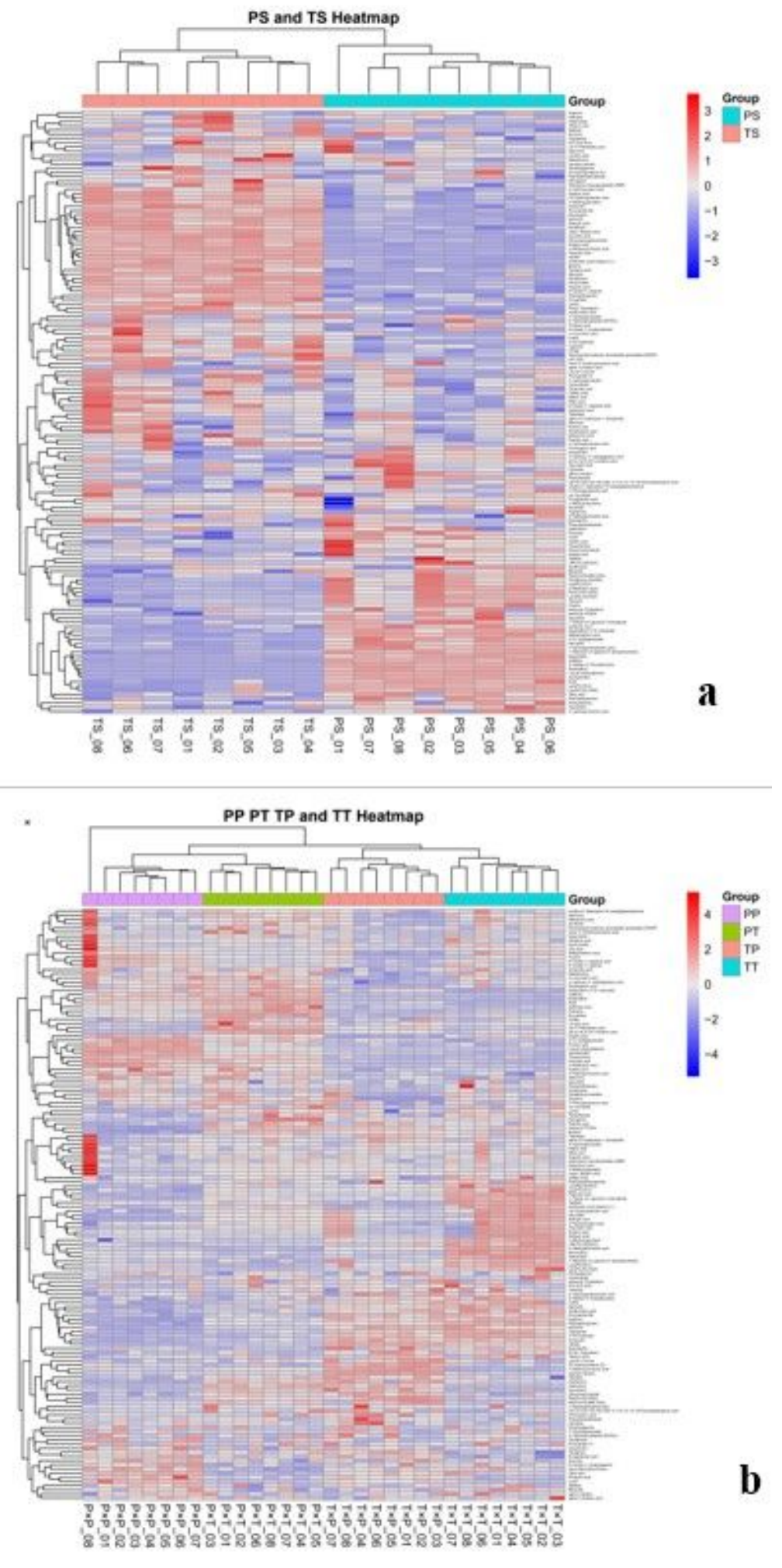

\section{Figure 6}

Heatmaps of different pollinations in $P S, T S(a)$ and $P \times P, P \times T, T \times T, T \times P(b)$. 

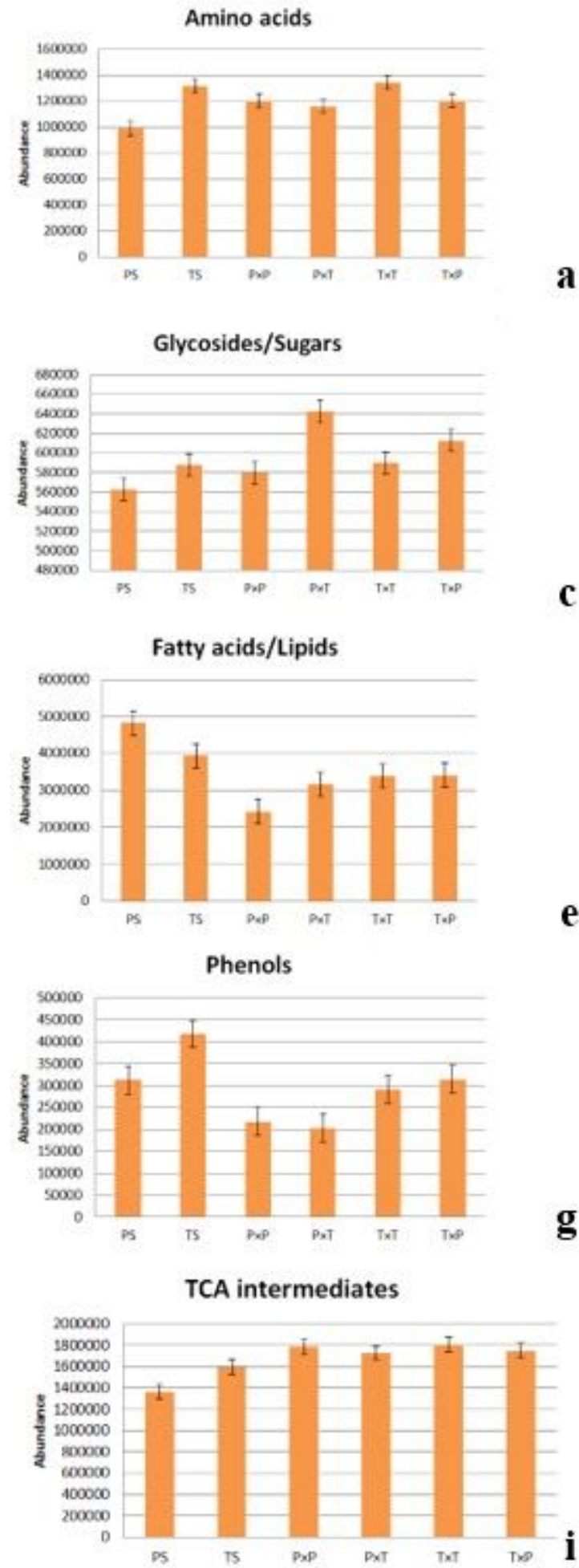
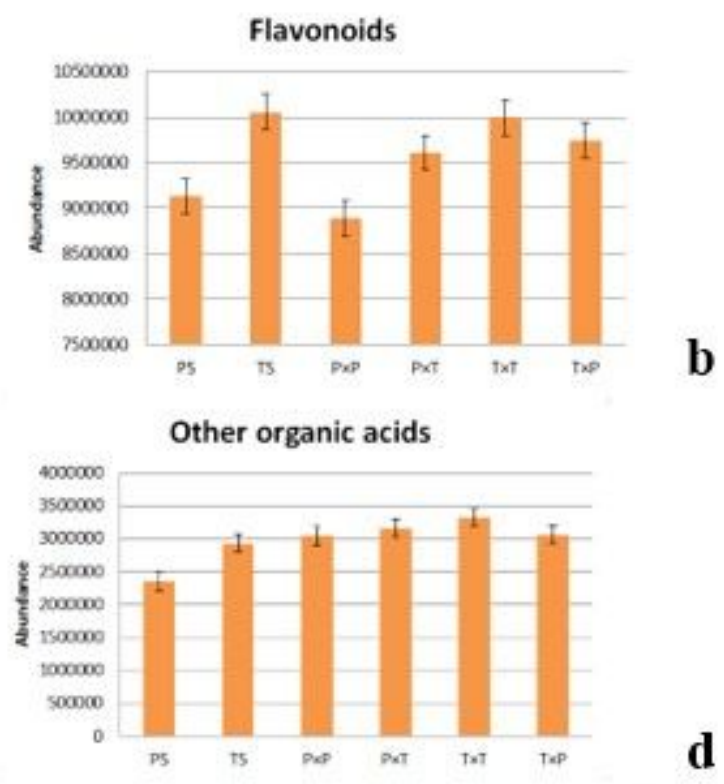

d
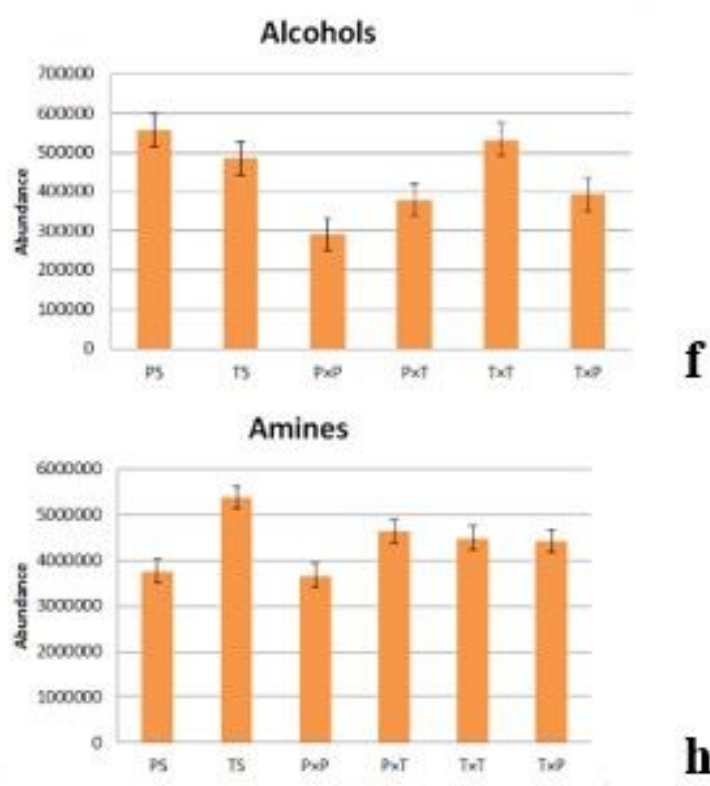

\section{Figure 7}

Histograms showing differences in metabolite contents in different pollinations: (a) amino acids; (b) flavonoids; (c) glycosides/sugars; (d) other organic compounds; (e) fatty acids/lipids; (f) alcohols; (g) phenols; (h) amines; (i) TCA intermediates.

\section{Supplementary Files}


This is a list of supplementary files associated with this preprint. Click to download.

- Glycosidesandsugars.xIsx

- TCA.xlsx

- Otherorganicacids.xlsx

- Aminoacids.xlsx

- Phenols.xisx

- Flavonoids.xlsx

- Alcohols.xIsx

- TableS2.xIsx

- TableS1.xlsx

- Fattyacidsandlipids.xlsx

- TableS4.xIsx

- TableS3.xlsx

- Amines.xlsx 